\title{
PROPUESTA DE RÚBRICA DE EVALUACIÓN PARA FORTALECER LOS PROCESOS CREATIVOS EN EL AULA
}

\author{
Sol Gómez \\ Catalina Huilcapi
}

\begin{abstract}
Resumen:
El presente artículo parte de la reflexión sobre la evaluación de los procesos creativos y propone la realización de una rúbrica genérica con diversos parámetros que pueden aportar en el proceso de evaluación de las áreas y asignaturas donde la creatividad está presente. La investigación identifica las herramientas de evaluación utilizadas en diferentes asignaturas de las Carreras de Artes Visuales y Diseño Gráfico y Comunicación Visual de la Pontificia Universidad Católica del Ecuador, donde se establece mayor uso de la creatividad según los estudiantes. Se analiza cómo la evaluación incide en la motivación de los educandos y en un proceso de enseñanza-aprendizaje significativo. Este estudio pretende ser un aporte para la práctica docente de profesores universitarios que acompañan este tipo de procesos y que esta propuesta pueda orientar a los educadores en sus procesos de evaluación.
\end{abstract}

Palabras claves: Evaluación educativa, proceso evaluativo, proceso creativo, instrumento de evaluación, rúbrica.

\begin{abstract}
:
This article examines assessment of creative activity and proposes grading parameters of diverse criteria that could contribute to the grading process in areas that develop creativity. This research point evaluation tools used in various classes offered by the Programs of Visual Arts and Design and Visual Communication at the Pontificia Universidad Católica del Ecuador, where according to students, there is an increased use of creativity. The study also observes how influential is evaluation for student motivation and teaching-learning process. This text looks forward to contributing to university professors' teaching practices who handle this kind of education and as well, intends to provide guidance on professors' evaluation processes.
\end{abstract}

Keywords: educational assessment, evaluation process, creative process, assessment tool, grading parameter.

\section{Autores:}

Sol Gómez (Quito, 1979). Graduada en Artes Plásticas en la Facultad de Artes de la Universidad Central del Ecuador, con especialización en Escultura y Grabado. Magíster en Ciencias de la Educación por la Pontificia Universidad Católica del Ecuador.

Catalina Huilcapi Collantes (Quito, 1978). Diseñadora y Magíster en Ciencias de la Educación por la Pontificia Universidad Católica del Ecuador. En este momento cursa una Especialización Superior en Educación y Nuevas Tecnologías en la Universidad Andina Simón Bolívar, Ecuador. 


\section{INTRODUCCIÓN}

Cuando se planteó este estudio fueron varias las preguntas que lo motivaron, por ejemplo: ¿cómo evaluar un proyecto en los campos de las artes y el diseño?, ¿existe una fórmula determinada para hacerlo?, ¿qué parámetros son los más acordes para evaluar el desarrollo de un proyecto? También, aportaron algunas inquietudes percibidas en los profesionales que se dedican a la docencia en estas áreas, en torno a las herramientas de evaluación que existen: ¿cuáles son y cómo utilizarlas para evaluar el desarrollo de un proyecto en el aula? Por último, se tomó en cuenta que en el documento curricular de las carreras de Artes Visuales y Diseño Gráfico y Comunicación Visual de la PUCE, no se establece con precisión qué técnicas o herramientas se deben utilizar para la evaluación de los aprendizajes.

Es imprescindible empezar con la postura que guía este estudio, que concibe a la educación como un espacio de compartir, de dar y recibir, de jugar y respetar, donde el intercambio de información, conocimientos, experiencias, valores y principios se realizan a través de la participación activa y colaborativa de cada uno de los actores del proceso educativo. Se entiende a la educación como un espacio flexible, diverso y de crecimiento, de respeto a las diferencias e individualidades, donde se permite tomar decisiones para la formación.

Desde esta postura, se ratifica un enfoque de la evaluación como un espacio de diálogo, de enriquecimiento mutuo y como un estado necesario para el constante mejoramiento, donde se sitúa en primer lugar a la persona, entendida como un ser integral ${ }^{1}$.

1 Hablar de un ser integral es comprender al ser humano en todo lo que le constituye, desde la parte cognitiva, pasando por
Sanmartí (2007) menciona que "evaluar es una condición necesaria para mejorar la enseñanza” pág. 123, ya que sea cual sea el ámbito es necesario ser parte de un proceso donde se encuentran aciertos y desaciertos, que permitan tomar decisiones para cambiar. La educación y por ende, la evaluación, no son estáticas sino cambiantes y con la posibilidad de innovar y recrear constantemente.

\section{OBJETIVOS}

- Reflexionar sobre la evaluación educativa como una experiencia que permite mejorar los procesos de enseñanza-aprendizaje en el aula.

- Evidenciar a través de una encuesta cómo los estudiantes se sienten en relación con la evaluación de sus proyectos.

- Dar pautas para la elaboración de instrumentos de evaluación de proyectos en los campos de las artes y el diseño, tales como una rúbrica para que pueda ser utilizada de manera genérica.

\section{EVALUAR NO ES MEDIR}

La evaluación de los aprendizajes es sin duda uno de los procesos más significativos por los que debe transitar un estudiante a lo largo de su formación, pues suscita experiencias positivas y negativas que impactan crucialmente en su disponibilidad para el aprendizaje y por ende, en su rendimiento académico y desarrollo integral. De aquí que evaluar es una actividad primordial

el área de las emociones y sensaciones, así como su aspecto físico, espiritual y social. 
dentro del hecho educativo, pues permite que el docente tome decisiones para mejorar las actividades de enseñanza y logre que los estudiantes alcancen el aprendizaje planificado. La información que se obtiene de la evaluación faculta al docente para emitir un juicio de valor sobre el aprendizaje de sus estudiantes, el mismo que constituye una retroalimentación necesaria que les permite verificar sus progresos e intervenir responsablemente en la mejora de su rendimiento. Además, el profesor debe usar esta información para mejorar su práctica docente y replantear las actividades de enseñanzaaprendizaje en caso que no estén suscitando experiencias de aprendizaje significativas. Por esto, se la debe realizar de manera continua y no solo al final del curso como única apreciación de los resultados, pues no es una actividad aislada ni la única medición obligatoria que señala la aprobación o reprobación de determinada asignatura.

Por todo lo dicho, evaluar no es sinónimo de medir o calificar. Cuando se mide o califica no se emite un juicio de valor, sino que se decide la nota que merece el estudiante con el fin de promoverlo o no. El profesor aplica criterios propios, no siempre objetivos, que en su mayoría no le sirven de guía al educando. Puede utilizar como base para medir los requerimientos del trabajo o proyecto que ha enviado con el fin de colocar una calificación que refleje el cumplimiento, pero esto tampoco lo orienta para alcanzar los resultados de aprendizaje deseados. Se deben proporcionar al estudiante instrumentos objetivos que reflejen cómo serán evaluados en función del aprendizaje requerido para dicho curso.

Cuando se prepara una evaluación es vital evitar la ejecución de prácticas aisladas y descontextualizadas, como la prueba objetiva «herramienta para la cual el docente redacta un número determinado de preguntas y al aplicarla, espera respuestas en su mayoría memorísticas». La calificación es alta si la respuesta refleja precisión literal con respecto a la fuente de donde se obtuvo la información. Es en este tipo de mediciones mal identificadas como evaluaciones, donde para el estudiante empieza una experiencia de cuantía de su capacidad para memorizar, para cumplir y para resistir a la cantidad de trabajo. Esta experiencia inmediatamente se confunde con una evaluación y se queda en el recuerdo del educando como única verdad acerca de este proceso. Quizás esta es una de las razones por las que los estudiantes no se implican en esta experiencia de evaluación, pues nunca esperan un juicio de valor constructivo o una retroalimentación positiva que los motive a alcanzar un aprendizaje permanentemente.

\section{PROCESO VERSUS RESULTADO}

La evaluación es una oportunidad de crecimiento y de visualización de los aciertos y desaciertos de cada uno de los actores del proceso educativo con el fin de continuar mejorando, en donde cada paso, cada encuentro y cada error son oportunidades de aprendizaje. En el proceso de concepción y elaboración de un proyecto, como en todos los procesos educativos, el desarrollo es fundamental, no solo porque no se puede evaluar una sola instancia «un fragmento de todo un camino recorrido" sino porque el proceso es el desarrollo mismo donde se puede ir viendo día a día el crecimiento de un estudiante en relación con su aprendizaje. Por tal razón, es esencial una evaluación continua y formadora, "la Evaluación Formadora es una estrategia de 
evaluación dirigida a promover la autorreflexión y el control sobre el propio aprendizaje" (López, 2009: 34). Este tipo de evaluación permite que el estudiante tome conciencia de su manera de aprender y de las acciones que fortalecen su propio proceso. Es importante no evaluar solo al resultado, más aún en el campo específico de las artes, pues se pierde un inmenso abanico de posibilidades y aprendizajes que van ocurriendo en la práctica diaria y que solo son posibles el momento de la reflexión y crítica constructiva que puede darse de manera personal, pero también de forma colectiva. En la evaluación del proceso es de suma importancia vincular el trabajo colaborativo, permitir espacios para la coevaluación. "La evaluación no tiene por qué estar exclusivamente en manos del enseñante. Cuando los estudiantes intervienen en el proceso, la oportunidad de evaluar se convierte en medio para promover su propia educación" (Eisner, 2011: 237).Así como la responsabilidad de empezar a emitir juicios de valor y de asumir estos criterios con fundamento y compromiso. Estos aspectos son fundamentales en las áreas donde la generación de nuevas ideas o conceptos y la autonomía, entre otras, son competencias básicas de carreras como las Artes Visuales, el Diseño Gráfico y la Comunicación Visual, pues al salir de los espacios de formación su capacidad de autocrítica y motivación son fundamentales para asegurar un desarrollo pleno de su profesión. "Mi opinión es que asumir parte de la responsabilidad de la evaluación es algo que los alumnos deben aprender, porque cuando estén fuera de la escuela no habrá ningún enseñante a su lado que lo haga por ellos. Llegar a la madurez significa hacerse independiente" (Eisner, 2011: 238).

\section{METODOLOGÍA}

La investigación realizada es de carácter cuantitativo y cualitativo. Para la investigación cuantitativa, se realizó un estudio preliminar con una muestra de 30 estudiantes que están cursando las Carreras de Artes Visuales y Diseño Gráfico y Comunicación Visual de la Facultad de Arquitectura, Diseño y Artes de la PUCE, tomando como referente su paso por primer semestre en las respectivas carreras, pues esto constituye su primer encuentro con la evaluación de los procesos de enseñanza-aprendizaje en nivel superior según los diferentes planes de estudio. Es importante recalcar que en las mencionadas carreras los cursos de primer nivel no sobrepasan los 15 estudiantes por paralelo. En Artes Visuales existe un paralelo, mientras que en Diseño generalmente dos.

Para la investigación cualitativa y debido a los resultados arrojados en semestres anteriores se tomó como referencia la evaluación de desempeño docente que se aplica en la Carrera de Diseño Gráfico y Comunicación Visual, la cual permite verificar varios procesos utilizados por los profesores, entre ellos los de evaluación. La herramienta que se utiliza es una encuesta con preguntas abiertas donde los estudiantes deben mencionar las fortalezas y debilidades del proceso evaluativo.

\subsection{RECOPILACIÓN DE INFORMACIÓN Y ANÁLISIS}

Se realizó una encuesta con nueve preguntas para indagar sobre la evaluación del desarrollo de un proyecto, tomando en cuenta aspectos como el proceso creativo, las herramientas que son utilizadas para dichas evaluaciones y sobre todo, para percibir cómo los estudiantes se sienten en relación con los instrumentos evaluativos que 
los docentes han utilizado, con el fin de reconocer las falencias y proponer otras herramientas para fomentar una evaluación formadora, clara y objetiva que potencialice los procesos de enseńanzaaprendizaje en el aula.

A continuación se detallan los resultados arrojados en dicha encuesta, los cuales se presentan en cuadros esquemáticos, con sus respectivos análisis:

\section{Cuadro 1. Instrumentos usados para evaluar el proceso creativo}

\begin{tabular}{|lcc|}
\hline Instrumento & $\mathbf{N}^{\circ}$ Estudiantes & Porcentaje \\
\hline Portafolio & 17 & $57 \%$ \\
Diario de trabajo & 16 & $53 \%$ \\
Criterios de evaluación & & \\
entregados por el docente & 14 & $47 \%$ \\
Pruebas orales & 6 & $20 \%$ \\
Rúbricas & 6 & $20 \%$ \\
Ensayos & 5 & $17 \%$ \\
Pruebas escritas & 2 & $7 \%$ \\
Listas de cotejo & 1 & $3 \%$ \\
Ninguno de los anteriores & 1 & $3 \%$ \\
No se utilizaron instrumentos & & \\
de evaluación claros y & & \\
objetivos & 1 & $3 \%$ \\
Organizadores gráficos & 0 & $0 \%$ \\
\hline
\end{tabular}

Fuente: Encuesta realizada a estudiantes por las autoras

Como se puede apreciar en el Cuadro 1, el portafolio es la herramienta de evaluación más utilizada por los docentes para evaluar el proceso creativo de un proyecto. Sin embargo, no difiere significativamente del manejo del diario de trabajo. Puede suceder que la herramienta no esté clara y exista una confusión entre los contenidos del portafolio, el diario de trabajo y una bitácora. Además surge la interrogante de cómo es evaluado el portafolio, cuáles son los criterios que se utilizan, así como los requerimientos en cuanto a contenidos y la objetividad con la cual se evalúa por medio de este instrumento. 


\section{Cuadro 2. Lo más valorado en una evaluación}

\begin{tabular}{|l|c|c|}
\hline \multicolumn{1}{|c|}{ Respuestas } & N $^{\circ}$ Estudiantes & Porcentaje \\
\hline $\begin{array}{l}\text { Que los comentarios } \\
\text { sean constructivos }\end{array}$ & 21 & $70 \%$ \\
\hline $\begin{array}{l}\text { Que me aporte al } \\
\text { aprendizaje }\end{array}$ & 16 & $53 \%$ \\
\hline $\begin{array}{l}\text { Que la evaluación sea } \\
\text { objetiva }\end{array}$ & 12 & $40 \%$ \\
\hline Que me motiven & 9 & $30 \%$ \\
\hline Otros & 0 & $0 \%$ \\
\hline
\end{tabular}

Fuente: Encuesta realizada a estudiantes por las autoras

En el Cuadro 2 es posible observar respuestas bastante significativas para este estudio. En primer lugar se aprecia que para los estudiantes, en una evaluación, la valoración de los comentarios constructivos que el profesor o el equipo evaluador emita acerca de los trabajos o proyectos es lo más importante. Seguidamente, se observa que los estudiantes valoran el aporte que la evaluación significa para el aprendizaje. Estos datos están relacionados profundamente con el significado de evaluación mencionado en este trabajo, pues los comentarios constructivos aportan al aprendizaje y transforman la experiencia de evaluación en un hecho positivo.

\section{Cuadro 3. Lo que ha generado experiencias negativas}

\section{en los procesos de evaluación}

\begin{tabular}{|l|c|c|}
\hline \multicolumn{1}{|c|}{ Respuestas } & N Estudiantes $^{\circ}$ & Porcentaje \\
\hline $\begin{array}{l}\text { Que el docente no realice } \\
\text { comentarios que aporten a } \\
\text { mi proceso }\end{array}$ & 12 & $40 \%$ \\
\hline $\begin{array}{l}\text { Que no conozco los } \\
\text { criterios de evaluación }\end{array}$ & 11 & $37 \%$ \\
\hline $\begin{array}{l}\text { Que no hay claridad en los } \\
\text { requerimientos }\end{array}$ & 9 & $30 \%$ \\
\hline $\begin{array}{l}\text { Que se evalúa solo el } \\
\text { resultado }\end{array}$ & 5 & $17 \%$ \\
\hline $\begin{array}{l}\text { Que no se individualiza el } \\
\text { proceso de evaluación }\end{array}$ & 4 & $13 \%$ \\
\hline $\begin{array}{l}\text { Que no siento apoyo } \\
\text { emocional }\end{array}$ & 8 & $27 \%$ \\
\hline Otros & & \\
\hline
\end{tabular}

Fuente: Encuesta realizada a estudiantes por las autoras 
En el Cuadro 3 se evidencia que las dos situaciones que han generado experiencias negativas son, en primer lugar aquellas donde el docente no realiza comentarios que aportan al proceso de aprendizaje, estrechamente seguidas por el hecho de no conocer los criterios usados para evaluar los trabajos o proyectos. Es evidente que el desconocimiento de los criterios, la ausencia de herramientas y de juicios de valor en el proceso de evaluación determinan el tipo de experiencia que se genera en el estudiante.

Otras preguntas que se realizaron requerían una respuesta cerrada con el fin de conocer si se realizan procesos de autoevaluación y coevaluación, los resultados fueron significativamente positivos. En la aplicación de la autoevaluación en el proceso creativo de un proyecto y de coevaluación, el 77\% de estudiantes respondió afirmativamente. Esto demuestra que hay aciertos en las prácticas de evaluación al involucrar al estudiante y a sus pares en la evaluación. Sin embargo, todavía queda la duda sobre los criterios que se utilizan para realizar estos procesos, si son objetivos y claros o están ligados a la relación de amistad entre los estudiantes.

\section{PROPUESTA:}

El estudio realizado advierte la necesidad de reforzar la utilización de instrumentos de evaluación que permitan generar experiencias potencialmente significativas, con un enfoque más objetivo y que fortalezcan los procesos de enseñanza-aprendizaje en el desarrollo de un proyecto en los campos

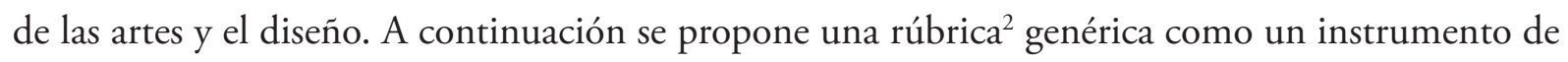
heteroevaluación, autoevaluación y coevaluación que puede ser utilizado para evaluar el desarrollo de un proyecto en el aula, así como para evaluar aspectos en la formación integral de los estudiantes. Esta herramienta no debe ser tomada como una camisa de fuerza, sino como un referente que puede ser modificado según las especificidades de cada materia y las necesidades de cada uno de los actores educativos.

2 Una rúbrica es un instrumento de evaluación que utiliza "una escala, que puede ser cuantitativa, cualitativa o una mezcla de ambas, la cual califica los diferentes niveles de logro" (Esquivel, 2009: 136). Este instrumento presenta una descripción detallada en cada nivel de la escala, que sirve de guía para el estudiante y el profesor en este proceso de evaluación de los resultados de aprendizaje y las competencias por su claridad y objetividad. Es vital que la rúbrica sea socializada y discutida con los estudiantes, para que tengan un rol activo dentro de este proceso y entiendan cómo funciona este instrumento. A partir de la aplicación de la rúbrica es fundamental emitir un juicio de valor hacia el estudiante, esto servirá para realizar la retroalimentación necesaria en los procesos educativos. 
RÚBRICA GENÉRICA PARA EVALUAR EL DESARROLLO DE UN PROYECTO EN LOS CAMPOS DE LAS ARTES Y EL DISEÑO

\begin{tabular}{|c|c|c|c|c|}
\hline & \multicolumn{4}{|c|}{ ESCALA } \\
\hline & LOGRADO & AVANZADO & MEDIO & INICIAL \\
\hline & $\begin{array}{l}\text { Cumple el } 100 \% \text { de } \\
\text { los requisitos. }\end{array}$ & $\begin{array}{l}\text { Cumple el } 75 \% \text { de los } \\
\text { requisitos. }\end{array}$ & $\begin{array}{l}\text { Cumple el } 50 \% \text { de los } \\
\text { requisitos. }\end{array}$ & $\begin{array}{l}\text { Cumple con menos } \\
\text { del } 50 \% \text { de los } \\
\text { requisitos. }\end{array}$ \\
\hline $\begin{array}{l}\text { CONTENIDOS/ } \\
\text { RUBROS }\end{array}$ & 5 & 4 & 3 & 2 a 1 \\
\hline \multirow[b]{3}{*}{$\begin{array}{l}\text { EXPERIMENTACIÓN / } \\
\text { FLEXIBILIDAD }\end{array}$} & $\begin{array}{l}\text { Presenta nuevas } \\
\text { posibilidades en } \\
\text { la resolución del } \\
\text { producto, proyecto o } \\
\text { trabajo. }\end{array}$ & $\begin{array}{l}\text { Presenta algunas } \\
\text { posibilidades en } \\
\text { la resolución del } \\
\text { producto, proyecto o } \\
\text { trabajo. }\end{array}$ & $\begin{array}{l}\text { En la resolución del } \\
\text { producto, proyecto } \\
\text { o trabajo usa } \\
\text { posibilidades que lo } \\
\text { podrían resolver, sin } \\
\text { embargo requiere } \\
\text { desarrollo. }\end{array}$ & $\begin{array}{l}\text { En la resolución del } \\
\text { producto, proyecto o } \\
\text { trabajo no usa nuevas } \\
\text { posibilidades. La } \\
\text { solución es recurrente. }\end{array}$ \\
\hline & $\begin{array}{l}\text { Se evidencia un } \\
\text { considerable aporte y } \\
\text { tiene ideas novedosas. }\end{array}$ & $\begin{array}{l}\text { Se evidencia cierto } \\
\text { aporte y/o ideas } \\
\text { novedosas. }\end{array}$ & $\begin{array}{l}\text { Se evidencia poco } \\
\text { aporte y/o ideas } \\
\text { novedosas, requiere } \\
\text { desarrollo. }\end{array}$ & $\begin{array}{l}\text { No hay aporte ni } \\
\text { evidencia de ideas } \\
\text { novedosas. }\end{array}$ \\
\hline & $\begin{array}{l}\text { Existe total disposición } \\
\text { a experimentar } \\
\text { en su proyecto, } \\
\text { trabajar desde } \\
\text { otras perspectivas, } \\
\text { ser flexible y no } \\
\text { mantenerse en el } \\
\text { espacio "seguro". }\end{array}$ & $\begin{array}{l}\text { Existe disposición } \\
\text { a experimentar en } \\
\text { su proyecto. Intenta } \\
\text { trabajar desde } \\
\text { otras perspectivas, } \\
\text { es medianamente } \\
\text { flexible y procura } \\
\text { no mantenerse en el } \\
\text { espacio "seguro". }\end{array}$ & $\begin{array}{l}\text { Existe poca disposición } \\
\text { a experimentar en su } \\
\text { proyecto. Le cuesta } \\
\text { trabajar desde otras } \\
\text { perspectivas. }\end{array}$ & $\begin{array}{l}\text { No existe disposición } \\
\text { a experimentar en } \\
\text { su proyecto .No es } \\
\text { flexible y se mantiene } \\
\text { en el espacio "seguro". }\end{array}$ \\
\hline PERTINENCIA & $\begin{array}{l}\text { Muestra una total y } \\
\text { clara relación de los } \\
\text { conceptos y/o teorías } \\
\text { con la resolución } \\
\text { creativa del problema. } \\
\text { Responde totalmente } \\
\text { a la necesidad del } \\
\text { comitente o del } \\
\text { contexto. }\end{array}$ & $\begin{array}{l}\text { Muestra cierta relación } \\
\text { de los conceptos } \\
\text { y/o teorías con } \\
\text { la resolución del } \\
\text { problema. Responde } \\
\text { de cierta forma a } \\
\text { la necesidad del } \\
\text { comitente o del } \\
\text { contexto. }\end{array}$ & $\begin{array}{l}\text { En la resolución del } \\
\text { problema muestra } \\
\text { escasa } \\
\text { relación con los } \\
\text { conceptos y/o } \\
\text { teorías. Responde } \\
\text { escuetamente a } \\
\text { la necesidad del } \\
\text { comitente o del } \\
\text { contexto. Requiere } \\
\text { desarrollo. }\end{array}$ & $\begin{array}{l}\text { En la resolución del } \\
\text { problema no relaciona } \\
\text { los } \\
\text { conceptos ni las } \\
\text { teorías. No responde } \\
\text { a la necesidad del } \\
\text { comitente o del } \\
\text { contexto. }\end{array}$ \\
\hline
\end{tabular}




\begin{tabular}{|c|c|c|c|c|}
\hline COHERENCIA & $\begin{array}{l}\text { Existe total coherencia } \\
\text { y consistencia entre el } \\
\text { material gráfico y los } \\
\text { fundamentos teóricos } \\
\text { del proyecto. }\end{array}$ & $\begin{array}{l}\text { El material gráfico } \\
\text { y los fundamentos } \\
\text { teóricos del proyecto } \\
\text { son coherentes } \\
\text { pero necesitan más } \\
\text { consistencia. }\end{array}$ & $\begin{array}{l}\text { El material gráfico y } \\
\text { la idea del proyecto } \\
\text { son medianamente } \\
\text { coherentes y } \\
\text { consistentes. }\end{array}$ & $\begin{array}{l}\text { Existe poca coherencia } \\
\text { y consistencia entre el } \\
\text { material gráfico y los } \\
\text { fundamentos teóricos } \\
\text { del proyecto. Necesita } \\
\text { mejorar. }\end{array}$ \\
\hline $\begin{array}{l}\text { ASOCIACIÓN Y } \\
\text { APLICACIÓN DE } \\
\text { SABERES (OTRAS } \\
\text { ASIGNATURAS) }\end{array}$ & $\begin{array}{l}\text { Asocia satisfactoria y } \\
\text { coherentemente los } \\
\text { saberes obtenidos en } \\
\text { otras asignaturas o en } \\
\text { niveles previos en el } \\
\text { producto, proyecto o } \\
\text { trabajo realizado. }\end{array}$ & $\begin{array}{l}\text { Asocia los saberes } \\
\text { obtenidos en otras } \\
\text { asignaturas o en } \\
\text { niveles previos pero } \\
\text { requiere mayor } \\
\text { coherencia en el } \\
\text { producto, proyecto o } \\
\text { trabajo realizado. }\end{array}$ & $\begin{array}{l}\text { Asocia escuetamente } \\
\text { los saberes obtenidos } \\
\text { en otras asignaturas o } \\
\text { en niveles previos en el } \\
\text { producto, proyecto o } \\
\text { trabajo realizado. }\end{array}$ & $\begin{array}{l}\text { No existe asociación } \\
\text { de los saberes } \\
\text { obtenidos en otras } \\
\text { asignaturas o en } \\
\text { niveles previos en el } \\
\text { producto, proyecto o } \\
\text { trabajo realizado. }\end{array}$ \\
\hline $\begin{array}{l}\text { COMUNICACIÓN } \\
\text { AUDIOVISUAL }\end{array}$ & $\begin{array}{l}\text { Utiliza } \\
\text { equilibradamente los } \\
\text { textos, gráficos, videos, } \\
\text { cromática y efectos } \\
\text { con el fin de mejorar } \\
\text { la comunicación visual } \\
\text { y el entendimiento de } \\
\text { los contenidos. }\end{array}$ & $\begin{array}{l}\text { Utiliza gráficos, videos, } \\
\text { cromática y efectos } \\
\text { con el fin de mejorar } \\
\text { la comunicación visual } \\
\text { y el entendimiento } \\
\text { de los contenidos, sin } \\
\text { embargo uno o dos } \\
\text { elementos no han sido } \\
\text { bien utilizados. Por } \\
\text { ejemplo: los bloques } \\
\text { de texto son tan } \\
\text { extensos que producen } \\
\text { ruido visual. }\end{array}$ & $\begin{array}{l}\text { Utiliza cromática y } \\
\text { efectos para mejorar } \\
\text { el aspecto de la } \\
\text { presentación, sin } \\
\text { embargo más de tres } \\
\text { elementos no han sido } \\
\text { bien utilizados. Por } \\
\text { ejemplo no presenta } \\
\text { videos, usa pocos } \\
\text { gráficos y la mayoría } \\
\text { de los bloques de texto } \\
\text { son muy extensos lo } \\
\text { cual causa pérdida de } \\
\text { atención. }\end{array}$ & $\begin{array}{l}\text { Utiliza una cromática } \\
\text { inapropiada. Los } \\
\text { efectos de secuencia } \\
\text { distraen la atención. } \\
\text { No utiliza videos, usa } \\
\text { pocos gráficos y la } \\
\text { mayoría de los bloques } \\
\text { de texto son muy } \\
\text { extensos lo cual causa } \\
\text { pérdida de atención. }\end{array}$ \\
\hline \multirow{2}{*}{$\begin{array}{c}\text { INVESTIGACIÓN/ } \\
\text { TRABAJO } \\
\text { AUTÓNOMO }\end{array}$} & $\begin{array}{l}\text { En su producto, } \\
\text { proyecto o } \\
\text { trabajo presenta } \\
\text { una satisfactoria } \\
\text { ampliación de la } \\
\text { información y nuevos } \\
\text { aportes. }\end{array}$ & $\begin{array}{l}\text { En su producto, } \\
\text { proyecto o trabajo } \\
\text { presenta cierta } \\
\text { ampliación de la } \\
\text { información y nuevos } \\
\text { aportes. }\end{array}$ & $\begin{array}{l}\text { En su producto, } \\
\text { proyecto o trabajo } \\
\text { presenta escueta } \\
\text { ampliación de la } \\
\text { información y nuevos } \\
\text { aportes. }\end{array}$ & $\begin{array}{l}\text { En su producto, } \\
\text { proyecto o trabajo no } \\
\text { presenta ampliación } \\
\text { de la información y } \\
\text { nuevos aportes. }\end{array}$ \\
\hline & $\begin{array}{l}\text { Se evidencia un } \\
\text { remarcable trabajo } \\
\text { autónomo de } \\
\text { investigación. }\end{array}$ & $\begin{array}{l}\text { Se evidencia cierto } \\
\text { trabajo autónomo de } \\
\text { investigación. }\end{array}$ & $\begin{array}{l}\text { Se evidencia escaso } \\
\text { trabajo autónomo de } \\
\text { investigación. }\end{array}$ & $\begin{array}{l}\text { No se evidencia un } \\
\text { trabajo autónomo de } \\
\text { investigación. }\end{array}$ \\
\hline
\end{tabular}




\section{PARÁMETROS COMPLEMENTARIOS PARA UNA EVALUACIÓN INTEGRAL}

Se ha considerado pertinente incluir otros parámetros de evaluación transversales al desarrollo de proyectos con el fin de aportar en la formación integral del estudiante. Estos parámetros están relacionados con las competencias genéricas que se requiere alcanzar según los perfiles de egreso de las carreras.

\begin{tabular}{|c|c|c|c|c|}
\hline & $\begin{array}{l}\text { El estudiante es } \\
\text { absolutamente } \\
\text { responsable } \\
\text { en cuanto al } \\
\text { uso de talleres, } \\
\text { laboratorios o aulas. } \\
\text { Utiliza todos los } \\
\text { implementos de } \\
\text { seguridad industrial } \\
\text { y cuida el espacio. }\end{array}$ & $\begin{array}{l}\text { El estudiante es } \\
\text { responsable en } \\
\text { cuanto al uso de } \\
\text { talleres, laboratorios } \\
\text { o aulas. Utiliza } \\
\text { casi todos los } \\
\text { implementos de } \\
\text { seguridad industrial } \\
\text { y cuida el espacio. }\end{array}$ & $\begin{array}{l}\text { El estudiante es } \\
\text { medianamente } \\
\text { responsable } \\
\text { en cuanto al } \\
\text { uso de talleres, } \\
\text { laboratorios o aulas. } \\
\text { Utiliza algunos } \\
\text { implementos } \\
\text { de seguridad } \\
\text { industrial y cuida } \\
\text { medianamente el } \\
\text { espacio. }\end{array}$ & $\begin{array}{l}\text { El estudiante es } \\
\text { poco responsable } \\
\text { en cuanto al uso de } \\
\text { talleres, laboratorios } \\
\text { o aulas. Utiliza } \\
\text { pocos implementos } \\
\text { de seguridad } \\
\text { industrial y no cuida } \\
\text { el espacio. }\end{array}$ \\
\hline $\begin{array}{c}\text { RESPONSABILIDAD } \\
\text { SOCIAL Y } \\
\text { AMBIENTAL }\end{array}$ & $\begin{array}{l}\text { El producto, } \\
\text { proyecto o trabajo } \\
\text { presentado } \\
\text { evidencia un estudio } \\
\text { profundo del } \\
\text { impacto ambiental } \\
\text { del mismo y del } \\
\text { uso responsable } \\
\text { de recursos y } \\
\text { tecnologías. } \\
\text { Considera aspectos } \\
\text { como las } 3 \text { R: } \\
\text { reutilización, } \\
\text { reciclaje y } \\
\text { reducción. }\end{array}$ & $\begin{array}{l}\text { El producto, } \\
\text { proyecto o trabajo } \\
\text { presentado evidencia } \\
\text { cierto estudio del } \\
\text { impacto ambiental } \\
\text { del mismo y del } \\
\text { uso responsable } \\
\text { de recursos y } \\
\text { tecnologías. } \\
\text { Considera aspectos } \\
\text { como las } 3 \mathrm{R} \text { : } \\
\text { reutilización, } \\
\text { reciclaje y reducción } \\
\text { sin embargo uno o } \\
\text { dos no son viables. }\end{array}$ & $\begin{array}{l}\text { El producto, } \\
\text { proyecto o trabajo } \\
\text { presentado evidencia } \\
\text { escaso estudio del } \\
\text { impacto ambiental } \\
\text { del mismo y del } \\
\text { uso responsable } \\
\text { de recursos y } \\
\text { tecnologías. } \\
\text { Considera } \\
\text { escuetamente } \\
\text { aspectos como las } \\
3 \text { R: reutilización, } \\
\text { reciclaje y } \\
\text { reducción. }\end{array}$ & $\begin{array}{l}\text { El producto, } \\
\text { proyecto o trabajo } \\
\text { presentado no } \\
\text { evidencia ningún } \\
\text { estudio del impacto } \\
\text { ambiental del } \\
\text { mismo y del } \\
\text { uso responsable } \\
\text { de recursos y } \\
\text { tecnologías. } \\
\text { No considera } \\
\text { aspectos como las } \\
3 \text { R: reutilización, } \\
\text { reciclaje y } \\
\text { reducción. }\end{array}$ \\
\hline
\end{tabular}




\begin{tabular}{|c|c|c|c|c|}
\hline \multirow{2}{*}{$\begin{array}{l}\text { MANEJO DE LAS } \\
\text { NTIC }\end{array}$} & $\begin{array}{l}\text { Selecciona y/o usa } \\
\text { adecuadamente } \\
\text { las NTIC para } \\
\text { comunicar con } \\
\text { absoluta eficacia } \\
\text { y eficiencia } \\
\text { su producto, } \\
\text { proyecto o trabajo. }\end{array}$ & $\begin{array}{l}\text { Selecciona y/o } \\
\text { usa las NTIC } \\
\text { para comunicar } \\
\text { su producto, } \\
\text { proyecto o trabajo } \\
\text { con cierta eficacia } \\
\text { y eficiencia. }\end{array}$ & $\begin{array}{l}\text { Selecciona y/o } \\
\text { usa las NTIC } \\
\text { para comunicar } \\
\text { su producto, } \\
\text { proyecto o trabajo; } \\
\text { sin embargo, estas } \\
\text { no constituyen } \\
\text { un apoyo total } \\
\text { al proceso de } \\
\text { comunicación del } \\
\text { mismo. Requiere } \\
\text { desarrollo. }\end{array}$ & $\begin{array}{l}\text { No selecciona } \\
\text { y/o usa } \\
\text { adecuadamente } \\
\text { las NTIC para } \\
\text { comunicar con } \\
\text { eficacia y eficiencia } \\
\text { su producto, } \\
\text { proyecto o trabajo. }\end{array}$ \\
\hline & $\begin{array}{l}\text { La incorporación } \\
\text { de las } \\
\text { herramientas } \\
\text { tecnológicas } \\
\text { potencian } \\
\text { absolutamente la } \\
\text { presentación y/o } \\
\text { comunicación de } \\
\text { sus productos, } \\
\text { proyectos o } \\
\text { trabajos. }\end{array}$ & $\begin{array}{l}\text { La incorporación } \\
\text { de las } \\
\text { herramientas } \\
\text { tecnológicas } \\
\text { aportan en la } \\
\text { presentación y/o } \\
\text { comunicación de } \\
\text { sus productos, } \\
\text { proyectos o } \\
\text { trabajos; sin } \\
\text { embargo, no } \\
\text { constituyen un } \\
\text { apoyo potencial. }\end{array}$ & $\begin{array}{l}\text { La incorporación } \\
\text { de las } \\
\text { herramientas } \\
\text { tecnológicas } \\
\text { potencian } \\
\text { y/o aportan } \\
\text { escuetamente la } \\
\text { presentación y/o } \\
\text { comunicación de } \\
\text { sus productos, } \\
\text { proyectos o } \\
\text { trabajos. }\end{array}$ & $\begin{array}{l}\text { La incorporación } \\
\text { de las } \\
\text { herramientas } \\
\text { tecnológicas } \\
\text { interfieren en la } \\
\text { presentación / } \\
\text { comunicación de } \\
\text { sus productos, } \\
\text { proyectos o } \\
\text { trabajos. }\end{array}$ \\
\hline \multirow[b]{2}{*}{$\begin{array}{l}\text { EXPOSICIÓN } \\
\text { GRUPAL / TRABAJO } \\
\text { EN EQUIPO }\end{array}$} & $\begin{array}{l}\text { Todo el contenido } \\
\text { es transmitido } \\
\text { equilibradamente } \\
\text { por los integrantes } \\
\text { del grupo. }\end{array}$ & $\begin{array}{l}\text { Casi todo el } \\
\text { contenido es } \\
\text { transmitido } \\
\text { equilibradamente } \\
\text { por los integrantes } \\
\text { del grupo. }\end{array}$ & $\begin{array}{l}\text { Al menos la mitad } \\
\text { del contenido } \\
\text { es transmitido } \\
\text { equilibradamente } \\
\text { por los integrantes } \\
\text { del grupo. }\end{array}$ & $\begin{array}{l}\text { Menos de la mitad } \\
\text { del contenido } \\
\text { es transmitido } \\
\text { equilibradamente } \\
\text { por los integrantes } \\
\text { del grupo. }\end{array}$ \\
\hline & $\begin{array}{l}\text { Se evidencia un } \\
\text { fuerte trabajo } \\
\text { en equipo. Los } \\
\text { integrantes del } \\
\text { grupo exponen } \\
\text { o explican con } \\
\text { solvencia el } \\
\text { trabajo o proyecto. } \\
\text { Existe absoluta } \\
\text { colaboración para } \\
\text { argumentarlo } \\
\text { y/o potenciar los } \\
\text { resultados. }\end{array}$ & $\begin{array}{l}\text { Se evidencia un } \\
\text { trabajo en equipo. } \\
\text { Los integrantes } \\
\text { del grupo logran } \\
\text { exponer o explicar } \\
\text { el trabajo o } \\
\text { proyecto. Existe } \\
\text { cierta colaboración } \\
\text { para argumentarlo } \\
\text { y/o potenciar los } \\
\text { resultados. }\end{array}$ & $\begin{array}{l}\text { Se evidencia poco } \\
\text { trabajo en equipo. } \\
\text { Los integrantes } \\
\text { del grupo exponen } \\
\text { o explican } \\
\text { escuetamente el } \\
\text { trabajo o proyecto. } \\
\text { Existe escasa } \\
\text { colaboración para } \\
\text { argumentarlo } \\
\text { y/o potenciar los } \\
\text { resultados. }\end{array}$ & $\begin{array}{l}\text { No se evidencia } \\
\text { un trabajo en } \\
\text { equipo. Los } \\
\text { integrantes del } \\
\text { grupo no exponen } \\
\text { o explican con } \\
\text { solvencia el trabajo } \\
\text { o proyecto. No } \\
\text { existe colaboración } \\
\text { para argumentarlo } \\
\text { y/o potenciar los } \\
\text { resultados. }\end{array}$ \\
\hline COMPROMISO & $\begin{array}{l}\text { El estudiante } \\
\text { demuestra total } \\
\text { compromiso con } \\
\text { su propio proyecto } \\
\text { o trabajo. Participa } \\
\text { activamente y } \\
\text { trae siempre } \\
\text { los materiales } \\
\text { solicitados. }\end{array}$ & $\begin{array}{l}\text { El estudiante } \\
\text { demuestra } \\
\text { compromiso con } \\
\text { su propio proyecto } \\
\text { o trabajo. Participa } \\
\text { con frecuencia y } \\
\text { trae los materiales } \\
\text { solicitados. }\end{array}$ & $\begin{array}{l}\text { El estudiante } \\
\text { demuestra } \\
\text { medianamente } \\
\text { compromiso } \\
\text { con su propio } \\
\text { proyecto o trabajo. } \\
\text { Participa poco y } \\
\text { trae algunos de } \\
\text { los materiales } \\
\text { solicitados. }\end{array}$ & $\begin{array}{l}\text { El estudiante } \\
\text { no demuestra } \\
\text { compromiso } \\
\text { con su propio } \\
\text { proyecto o trabajo. } \\
\text { No participa en } \\
\text { clase y no trae } \\
\text { los materiales } \\
\text { solicitados. }\end{array}$ \\
\hline
\end{tabular}




\begin{tabular}{|c|c|c|c|c|}
\hline EXPOSICIÓN ORAL & $\begin{array}{l}\text { La exposición oral } \\
\text { es completamente } \\
\text { clara, es elocuente, } \\
\text { utiliza palabras } \\
\text { propias y logra } \\
\text { argumentar su } \\
\text { proyecto. Usa } \\
\text { apuntes solo para } \\
\text { recordar ideas } \\
\text { claves. }\end{array}$ & $\begin{array}{l}\text { La exposición oral } \\
\text { es clara, se hace } \\
\text { entender, pero } \\
\text { falta argumentar } \\
\text { su proyecto. Casi } \\
\text { todo el contenido } \\
\text { es transmitido } \\
\text { con las palabras } \\
\text { propias. Usa } \\
\text { apuntes para } \\
\text { recordar los } \\
\text { contenidos } \\
\text { y en escasas } \\
\text { ocasiones los lee } \\
\text { textualmente. }\end{array}$ & $\begin{array}{l}\text { La exposición } \\
\text { oral se entiende } \\
\text { medianamente, } \\
\text { por lo que no se } \\
\text { comprende con } \\
\text { facilidad y los } \\
\text { argumentos no } \\
\text { son pertinentes. } \\
\text { Al menos la mitad } \\
\text { del contenido } \\
\text { es transmitido } \\
\text { con las palabras } \\
\text { propias del } \\
\text { estudiante. Usa } \\
\text { apuntes para } \\
\text { recordar los } \\
\text { contenidos y lee la } \\
\text { mitad del tiempo } \\
\text { en estos. }\end{array}$ & $\begin{array}{l}\text { Presenta } \\
\text { dificultades en } \\
\text { expresar y hacer } \\
\text { comprensible su } \\
\text { idea y no presenta } \\
\text { argumentos. } \\
\text { Menos de la mitad } \\
\text { del contenido } \\
\text { es transmitido } \\
\text { con las palabras } \\
\text { propias del } \\
\text { estudiante. . } \\
\text { Usa apuntes } \\
\text { para recordar los } \\
\text { contenidos y lee } \\
\text { todo el tiempo en } \\
\text { estos. }\end{array}$ \\
\hline $\begin{array}{c}\text { COMUNICACIÓN } \\
\text { ESCRITA }\end{array}$ & $\begin{array}{l}\text { No existen errores } \\
\text { ortográficos ni } \\
\text { gramaticales en el } \\
\text { proyecto o trabajo. }\end{array}$ & $\begin{array}{l}\text { Existe } 1-2 \text { errores } \\
\text { ortográficos y/o } \\
\text { gramaticales en el } \\
\text { proyecto o trabajo. }\end{array}$ & $\begin{array}{l}\text { Existen } 3-4 \\
\text { errores de tipo } \\
\text { ortográfico y/o } \\
\text { gramatical en } \\
\text { el proyecto o } \\
\text { trabajo, aunque } \\
\text { no interfiere en la } \\
\text { lectura. }\end{array}$ & $\begin{array}{l}\text { Existen más } \\
\text { de } 5 \text { errores } \\
\text { gramaticales y } \\
\text { ortográficos en el } \\
\text { proyecto o trabajo } \\
\text { que distrae la } \\
\text { atención del lector } \\
\text { y distorsiona el } \\
\text { contenido. }\end{array}$ \\
\hline $\begin{array}{c}\text { SÍNTESIS ORAL Y } \\
\text { ESCRITA }\end{array}$ & $\begin{array}{l}\text { Demuestra gran } \\
\text { síntesis de la } \\
\text { información } \\
\text { escrita de su } \\
\text { proyecto, } \\
\text { producto o } \\
\text { trabajo. Comunica } \\
\text { de manera clara y } \\
\text { concreta la idea. }\end{array}$ & $\begin{array}{l}\text { Demuestra cierto } \\
\text { grado de síntesis } \\
\text { en la información } \\
\text { sobre su proyecto, } \\
\text { producto o } \\
\text { trabajo. Comunica } \\
\text { medianamente la } \\
\text { idea. }\end{array}$ & $\begin{array}{l}\text { Demuestra poca } \\
\text { síntesis en la } \\
\text { información } \\
\text { sobre su proyecto, } \\
\text { producto o } \\
\text { trabajo. Comunica } \\
\text { confusamente la } \\
\text { idea. }\end{array}$ & $\begin{array}{l}\text { No demuestra } \\
\text { síntesis en la } \\
\text { información } \\
\text { sobre su proyecto, } \\
\text { producto o } \\
\text { trabajo. No } \\
\text { comunica la idea } \\
\text { de manera clara y } \\
\text { concreta. }\end{array}$ \\
\hline
\end{tabular}

Este instrumento puede ser utilizado de diversas maneras, según el trabajo o proyecto a evaluar, por ejemplo, se puede aumentar en la misma rúbrica la calificación a la que corresponde cada uno de los criterios como se puede apreciar en el siguiente cuadro:

\begin{tabular}{|c|c|c|c|c|}
\hline EXPOSICIÓN ORAL & $\begin{array}{l}\text { La exposición oral } \\
\text { es completamente } \\
\text { clara, se hace } \\
\text { entender con } \\
\text { facilidad y logra } \\
\text { argumentar } \\
\text { su proyecto. } \\
\text { 2ptos. }\end{array}$ & $\begin{array}{l}\text { La exposición oral } \\
\text { es clara, se hace } \\
\text { entender, pero } \\
\text { falta argumentar } \\
\text { su proyecto. } \\
\mathbf{1 , 5 \text { ptos. }}\end{array}$ & $\begin{array}{l}\text { La exposición } \\
\text { oral se entiende } \\
\text { medianamente, } \\
\text { por lo que no se } \\
\text { comprende con } \\
\text { facilidad y los } \\
\text { argumentos no } \\
\text { son pertinentes. } \\
\text { 1pto. }\end{array}$ & 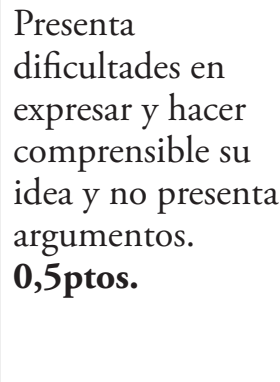 \\
\hline
\end{tabular}




\section{CONCLUSIONES}

Se pudo evidenciar que la utilización de rúbricas dentro del sistema de evaluación en los campos de las artes y el diseño es poco frecuente, seguramente esto se debe a que una gran parte de los docentes que trabajan en estos campos desconocen las técnicas y herramientas de evaluación que existen. Es fundamental generar espacios para compartir herramientas pedagógicas que ayuden en el mejoramiento de los procesos de enseñanza-aprendizaje como lo es la evaluación.

Asimismo, es imprescindible que el profesor planifique los programas microcurriculares incluyendo claramente las técnicas, los parámetros y los instrumentos de evaluación que serán utilizados para controlar los logros obtenidos a lo largo de todo el proceso, esto será beneficioso para el estudiante y para el mismo docente.

Los beneficios de una evaluación procesual, continua y formadora son positivos pues ayudan a mejorar la motivación e implicación del estudiante en el proceso de aprendizaje, tanto individual como colectivo. Fomentan el crecimiento, la independencia en el área de formación y en el aspecto personal, además permiten evidenciar los desaciertos para ir tomando correctivos.

Cuando el profesor permite que el estudiante se involucre en la elaboración de herramientas de evaluación o cuando socializa las mismas hasta alcanzar su comprensión, ayuda al educando a la construcción de procesos mentales complejos que fomentan el desarrollo creativo y el pensamiento crítico frente a su propio proceso de aprendizaje y desarrollo integral.

Por último, diseñar una rúbrica genérica para que los actores del proceso educativo puedan utilizarla como un referente tanto para evaluar el desarrollo de un proyecto en los campos de las artes y el diseño como para la formación integral del alumno, ha permitido evidenciar que inclusive las áreas consideradas sensibles o subjetivas son evaluables con pautas claras y objetivas.

\section{Bibliografía}

Calero, M., (2012). Creatividad. Reto de innovación educativa. México: Alfaomega Grupo Editorial, S.A.

Castillo, S. y Cabrerizo, J., (2010). La práctica de la evaluación educativa - Materiales e instrumentos. Madrid: Pearson Educación, S.A.

Castillo, S. y Cabrerizo, J., (2010). Evaluación educativa de aprendizajes y competencias. Madrid: Pearson Educación, S.A.

Delors, J., (1996). Los Cuatro Pilares de la Educación en La Educación Encierra un Tesoro (pp.89-103). Madrid: UNESCO.

Eisner, E., (2011). Elarte y la creación de la mente. El papel de las artes visuales en la transformación de la conciencia. Barcelona: Paidós.

Errázuris, L. H.. (2002). Cómo Evaluar el Arte. Santiago de Chile: Fontaine Editores. Recuperado el 13 de octubre de 2015, de: http://www.memoriachilena.cl/archivos2/pdfs/ MC0054999.pdf

Esquivel, J. M., (2009). Evaluación de los aprendizajes en el aula: una conceptualización renovada. En Martín, E. y Martínez, F. (Coord.). Avances y desafíos en la evaluación educativa. (pp. 127-143). OEI. Fundación Santillana. 
García, J., (2000). Bases Pedagógicas de la Evaluación (Guía práctica para educadores). Madrid: Editorial Síntesis.

Huilcapi, C., (2013). Impacto del uso de las NTIC's en la cátedra tecnológico gráfico y comunicación visual III como apoyo al proceso de aprendizaje. Tesis de Maestría. Carrera de Ciencias de la Educación. PUCE. Quito.

López, V. (Coord.) (2009). Evaluación Formativa y Compartida en Educación Superior - Propuestas, técnicas, instrumentos y experiencias. Madrid: Narcea, S.A. de EDICIONES.

Morin, E., (1999). Los Siete Saberes Necesarios para la Educación del futuro. Santillana.

PUCE. (2012). Aprender a Aprender en la PUCE. Modelo Educativo para una Nueva Universidad. El Paradigma Pedagógico Ignaciano. Quito: PUCE.

Sanmartí, N., (2007). 10 ideas clave. Evaluar para aprender. Barcelona: Editorial Graó. 